Results A total of 22 doctors attended the sessions. Adherence to the overall standards improved from 58 in the $1^{\text {st }}$ session to $72 \%$ in the $2^{\text {nd }}$. An improvement was seen in six standards, compliance maintained in two and a fall in compliance noted in two standards. A further re-audit over a three week period found an improvement in these two deteriorated standards, with an overall adherence of $90 \%$. Feedback from the sessions was very positive with a suggestion for other audits to be carried out in a similar format. A three week audit at another paediatric unit within the same Trust where no similar training sessions were performed found adherence to the standards of $70 \%$.

Conclusion The introduction of a programme which utilised the prescribers themselves enabled a level of engagement and a greater understanding of the standards required. A significant improvement in prescribing was noted over the period of assessment which, following repeated sessions, is expected to be maintained.

\section{G331(P) HAEMOPHILIA: A LOT TO LEARN FROM A NEAR MISS EVENT}

${ }^{1} \mathrm{G}$ Bradley, ${ }^{1}$ A Mukherjee, ${ }^{2}$ AM Will. ${ }^{1}$ Paediatrics, Royal Oldham Hospital, Pennine Acute Hospitals NHS Trust, Oldham, UK; ${ }^{2}$ Paediatric Haematology, Royal Manchester Children's Hospital, Manchester, UK

\subsection{6/archdischild-2015-308599.307}

Introduction A two year old girl presented with prolonged bleeding following a fall. A strong family history of haemophilia was ignored during visits to health professionals due to the misconception that female carriers are unaffected! She was extensively investigated and was found to have Factor VIII deficiency which did not respond to Tranexamic acid and Desmopressin. This was discovered three weeks prior to her preoperative assessment for an adenotonsillectomy. Routine pre op checks, which do not always involve clotting and the above misconception, could have affected her severely perioperatively. This case highlights the importance of detailed history taking including family history and remembering that Haemophilia can, and does affect females.

Case report AG was born without complication, at term by forceps delivery. Mother had heavy postpartum bleeding. She developed obstructive sleep apnoea, and adeno-tonsillectomy was planned. Three weeks before her pre-operative assessment she fell, tearing her frenulum. Persistent bleeding led to a paediatric assessment. Initial investigation revealed her haemoglobin was 88, APTT 1.5 and APTT ratio 45 and rest normal. Further investigation revealed low Factor VIIIc levels (36), 72\% of expected. Other factors were normal. Detailed history taking revealed a family history of Factor VIII deficiency (maternal grandmother: carrier, maternal aunt: affected). AG received Tranexamic Acid and a Desmopressin infusion, followed by a Factor VIII infusion at the Haematology unit due to persistent bleeding. Communications between ENT a haematology is ongoing to carry out a safe surgery.

Conclusion This was a near miss event which will require ongoing care from a consultant haematologist. Clinicians need to be aware that Haemophilia can, and does, affect females. 28\% of female carriers of Haemophilia A are known to have Factor VIII levels consistent with mild Haemophilia. This case should raise our awareness about thorough history taking, and updating our knowledge about Haemophilia, to avoid future catastrophes. There are also important implications for her mother. We recommend checking Factor VIII activity levels in all haemophilia carriers before haemostatic challenge, such as pregnancy.

\section{G332(P) EXCESSIVE DAYTIME SLEEPINESS IN TEENAGERS - COULD IT BE DUE TO IRON DEFICIENCY?}

C Kallappa, T Ninan. Paediatric, Heart of England NHS Hospitals Foundation Trust, Birmingham, UK

\subsection{6/archdischild-2015-308599.308}

Introduction Research has shown that restless leg syndrome, restless sleep or insomnia among paediatric population is often related to low iron stores. Dopamine plays a role in neuronal networks including sleep activity. Iron is vital to the brain's dopamine system and therefore iron deficiency is a contributing factor to sleep disorders. $1.9 \%$ of children and $2 \%$ of adolescents are affected with restless leg syndrome ${ }^{1}$ A 2002 study showed that abnormal iron stores or metabolism may result in restless leg syndrome causing insomnia in teenagers. ${ }^{2}$

Back ground To assess teenagers presenting with recent onset sleep problems with respect to presenting complaints, RLS (restless leg syndrome) questionnaire, investigations and measuring outcomes using Modified Paediatric Epworth Sleepiness Scale (Ages 6-16), pre and post treatment.

Patients and methods 5 teenagers aged between 14 and 15 years, presenting with excessive day time sleepiness and tiredness referred to sleep clinic in the last 12 months were analysed. They were sent sleep questionnaire prior to clinic visit. They completed the RLS questionnaire in the clinic and the modified paediatric Epworth sleepiness scale. Those found to have Ferritin $<50$ microgram/lt were treated with iron supplements for 3 months along with sleep hygiene and dietary advice.

Results $3 / 5$ teenagers had nocturnal leg pains with multiple arousals. The RLS questionnaire was positive for all 5 questions. Epworth sleepiness score was $>10$. Iron studies showed a mean of $9 \mathrm{mmols} / \mathrm{lt}(10.7-28.6)$ and ferritin of $9.7 \mathrm{ng} / \mathrm{ml}(5-204)$ respectively. Post iron treatment, clinical symptoms and Epworth scoring improved (0-2).

Conclusion Sleep related symptoms in these patients were due to the restless legs. This supports the hypothesis that low iron levels may result in RLS causing sleep disturbances in teenagers. Iron studies including ferritin levels in teenagers is indicated with insomnia, excessive daytime sleepiness of unexplained origin even when anaemia is mild or absent.

We recommend commencing iron supplementation for patients with ferritin of $<50 \mathrm{ng} / \mathrm{lt}$ along with sleep hygiene and dietary advice.

\section{British Society for Paediatric and Adolescent Rheumatology and British Society of Paediatric Gastroenterology, Hepatology and Nutrition}

\section{G353 HAS THE RISING INCIDENCE OF PAEDIATRIC INFLAMMATORY BOWEL DISEASE IN OUR REGION STABILISED?}

L Selvarajan, HR Jenkins. University Hospital of Wales, Cardiff, UK

10.1136/archdischild-2015-308599.309 